\title{
Dielectric properties of ionomers at microwave frequencies
}

\author{
K.T. Mathew ${ }^{\mathrm{a}, *}$, S. Biju Kumar ${ }^{\mathrm{a}}$, Anil Lonappan ${ }^{\mathrm{a}}$, Joe Jacob ${ }^{\mathrm{a}}$, Jacob Samuel ${ }^{\mathrm{b}}$, \\ Thommachan Xavier ${ }^{\mathrm{b}}$, Thomas Kurian ${ }^{\mathrm{b}}$ \\ ${ }^{a}$ Department of Electronics, Cochin University of Science and Technology, Kochi-682 022, India \\ ${ }^{\mathrm{b}}$ Department of Polymer Science and Rubber Technology, Cochin University of Science and Technology, Kochi-682 022, India
}

Received 7 October 2001; accepted 15 November 2001

\begin{abstract}
Ionic polymers (ionomers) with interesting characteristics are emerging as important commercial polymers. Ionomers have the unique ability to behave as cross-linked materials at ambient temperatures and to melt and flow at elevated temperatures like thermoplastics. The complex permittivity and conductivity of a class of ionomers at microwave frequencies are determined using the cavity perturbation technique and the results are presented.
\end{abstract}

(C) 2002 Elsevier Science B.V. All rights reserved.

Keywords: Ionomers; Complex permittivity; Microwaves; Cavity perturbation technique

\section{Introduction}

Ionic polymers or ionomers basically contain a small percentage of ionic groups attached to a hydrocarbon backbone. The ionic interaction between the groups results to significant changes in polymer properties [1-3]. The ionomer has an intermediate position between purely organic and purely inorganic structures. Considering the thermally reversible behaviour and enhanced mechanical properties, it seems to be an ideal material for many applications.

Very little is known about the microwave properties of ionomers. Microwave study on the dielectric properties of these materials reveals some interesting observations. A detailed study on the complex per-

\footnotetext{
* Corresponding author. Tel.: +91-484-532161; fax: +91-484532800 .

E-mail address: mathew@doe.cusat.edu (K.T. Mathew).
}

mittivity, loss tangent and conductivity at four different frequencies in $\mathrm{S}$ band $(2-4 \mathrm{GHz})$ for the different ionomer samples is presented.

\section{Principle}

Cavity perturbation technique [4] is employed for the study. Closed section of a waveguide constitutes a waveguide cavity resonator. The cavity resonator can be of transmission or reflection type. Electromagnetic energy is coupled to the cavity through coupling holes at the ends of the cavity. A nonradiating slot is provided at the broad wall of the cavity for the introduction of the sample. The cavity resonates at different frequencies depending on its dimensions. The schematic diagram of the transmission type cavity is shown in Fig. 1.

The basic principle involved in the technique is that the field within the cavity resonator is perturbed by the introduction of the dielectric sample through the non- 


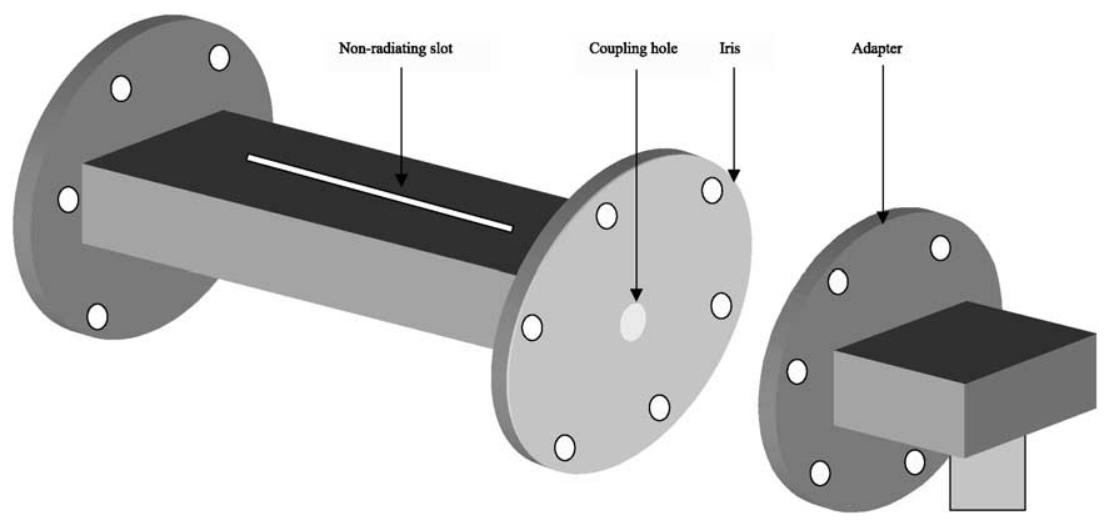

Fig. 1. Schematic diagram of the cavity resonator.

radiating slot. The resonant frequency and the quality factor of the cavity get shifted by the perturbation. The shift in the frequency is a measure of dielectric constant and that in quality factor gives the loss factor. The conductivity of the sample can be found out from the loss factor.

\section{Sample preparation}

Samples were prepared by sulphonation of highstyrene resin (HSR) using the sulphonating agent, acetyl sulphate, generated in situ, from acetic anhydride and concentrated sulphuric acid. The HSR sulphonic acid produced was neutralised by a solution of zinc acetate in methanol. The zinc-sulphonated HSR was washed several times with water and then it was vacuum-dried at $50{ }^{\circ} \mathrm{C}$. This ionic polymer is hereafter represented as $x y \mathrm{ZnS}-\mathrm{HSR}$, where $x y$ shows the number of milliequivalents of sulphonic acid per 100 $\mathrm{g}$ of HSR. Five samples were considered, they are $\mathrm{I}_{0}$, $\mathrm{I}_{10}, \mathrm{I}_{20}, \mathrm{I}_{30}$ and $\mathrm{I}_{40}$. $\mathrm{I}_{0}$ represents the pure HSR. $\mathrm{I}_{10}, \mathrm{I}_{20}$, $\mathrm{I}_{30}$ and $\mathrm{I}_{40}$ indicate 10.6, 20.4, 34.38 and 42.7 milliequivalents of sulphonate per $100 \mathrm{~g}$ of HSR. Quantity of sulphonate is analysed using X-ray fluorescence (XRF) method.

\section{Experimental set-up}

The experimental set-up consists of a transmission type S-band rectangular cavity resonator, HP 8714 ET network analyser and an interfacing computer as shown in Fig. 2. The cavity resonator is excited in the $\mathrm{TE}_{10 \mathrm{p}}$ mode. A typical resonant frequency spectrum of the cavity resonator is shown in Fig. 3. Initially, the resonant frequency $f_{\mathrm{o}}$ and the corresponding quality factor $Q_{\mathrm{o}}$ of each resonant peak of the empty cavity are

HP 8714 ET NETWORK ANALYSER

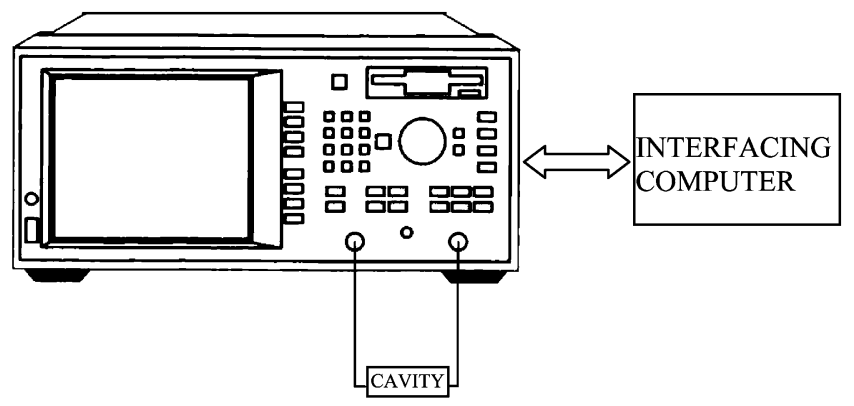

Fig. 2. Experimental set-up. 


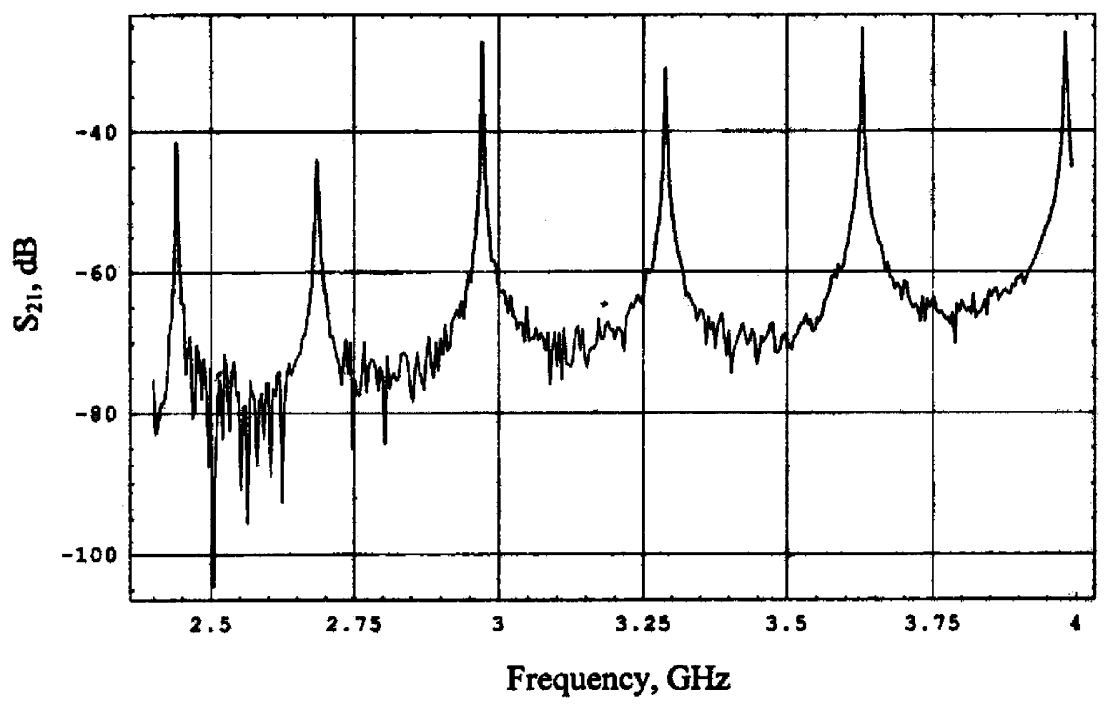

Fig. 3. Resonant frequency spectrum of cavity resonator.

determined. Samples prepared in the form of thin strip are introduced into the cavity resonator through the nonradiating slot. One of the resonant frequencies of the loaded cavity is selected and the position of the

Table 1

Dielectric properties of ionomer samples at different microwave frequencies

\begin{tabular}{llll}
\hline Frequency $(\mathrm{GHz})$ & Sample & $\varepsilon_{\mathrm{r}}^{\prime}-1$ & $\varepsilon_{\mathrm{r}}^{\prime \prime}$ \\
\hline 2.246 & $\mathrm{I}_{0}$ & 1.426 & 0.0026 \\
& $\mathrm{I}_{10}$ & 0.347 & 0.0281 \\
& $\mathrm{I}_{20}$ & 1.465 & 0.0576 \\
& $\mathrm{I}_{30}$ & 1.718 & 0.0594 \\
2.438 & $\mathrm{I}_{40}$ & 1.853 & 0.1281 \\
& $\mathrm{I}_{0}$ & 1.393 & 0.0084 \\
& $\mathrm{I}_{10}$ & 1.533 & 0.0369 \\
& $\mathrm{I}_{20}$ & 1.623 & 0.0624 \\
& $\mathrm{I}_{30}$ & 1.732 & 0.0694 \\
& $\mathrm{I}_{40}$ & 1.889 & 0.0980 \\
& $\mathrm{I}_{0}$ & 1.427 & 0.0129 \\
& $\mathrm{I}_{10}$ & 1.689 & 0.0394 \\
& $\mathrm{I}_{20}$ & 1.620 & 0.0657 \\
& $\mathrm{I}_{30}$ & 1.747 & 0.0704 \\
& $\mathrm{I}_{40}$ & 1.891 & 0.1007 \\
& $\mathrm{I}_{0}$ & 1.378 & 0.0084 \\
& $\mathrm{I}_{10}$ & 1.538 & 0.0384 \\
& $\mathrm{I}_{20}$ & 1.582 & 0.0637 \\
& $\mathrm{I}_{30}$ & 1.718 & 0.0714 \\
& $\mathrm{I}_{40}$ & 1.875 & 0.0962 \\
\hline
\end{tabular}

sample is adjusted for maximum perturbation (i.e. maximum shift of resonant frequency with minimum amplitude for the peak). The new resonant frequency $f_{\mathrm{s}}$ and 3-dB bandwidth and hence, the quality factor $Q_{\mathrm{s}}$ are determined. The procedure is repeated for other resonant frequencies.

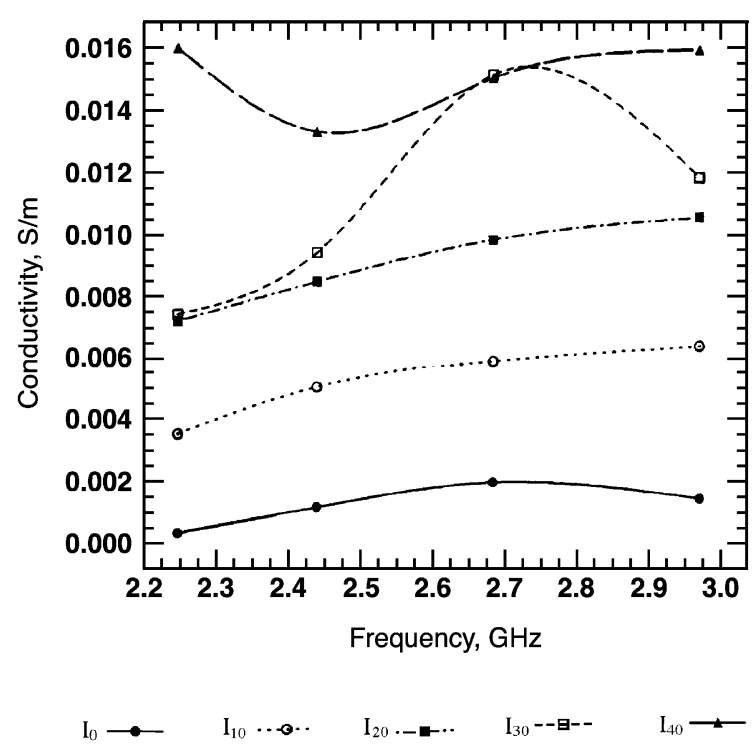

Fig. 4. Variation of conductivity of ionomers with frequency. 


\section{Theory}

According to the theory of cavity perturbation, the complex frequency shift is related as [5]:

$-\frac{\mathrm{d} \Omega}{\Omega} \approx \frac{\left(\overline{\varepsilon_{\mathrm{r}}}-1\right) \int_{V_{\mathrm{s}}} E E_{0}^{*} \mathrm{~d} V}{2 \int_{V_{\mathrm{c}}}\left|E_{0}\right|^{2} \mathrm{~d} V}$.

But,

$\frac{\mathrm{d} \Omega}{\Omega} \approx \frac{\mathrm{d} \omega}{\omega}+\frac{j}{2}\left[\frac{1}{Q_{\mathrm{s}}}-\frac{1}{Q_{0}}\right]$.

Equating (1) and (2) and separating real and imaginary parts, we get:

$\varepsilon_{\mathrm{r}}^{\prime} 1=\frac{f_{\mathrm{o}}-f_{\mathrm{s}}}{2 f_{\mathrm{s}}}\left(\frac{V_{\mathrm{c}}}{V_{\mathrm{s}}}\right)$

$\varepsilon_{\mathrm{r}}^{\prime \prime}=\frac{V_{\mathrm{c}}}{4 V_{\mathrm{s}}}\left(\frac{Q_{\mathrm{o}}-Q_{\mathrm{s}}}{Q_{\mathrm{o}} Q_{\mathrm{s}}}\right)$

Here, $\overline{\varepsilon_{\mathrm{r}}}=\varepsilon_{\mathrm{r}}^{\prime}-j \varepsilon_{\mathrm{r}}^{\prime \prime} ; \overline{\varepsilon_{r}}$ is the relative complex permittivity of the sample, $\varepsilon_{\mathrm{r}}^{\prime}$ is the real part of the relative complex permittivity, which is usually known as dielectric constant, and $\varepsilon_{\mathrm{r}}^{\prime \prime}$ is the imaginary part of the relative complex permittivity, which is associated with the dielectric loss of the material. $V_{\mathrm{s}}$ and $V_{\mathrm{c}}$ are the volumes of the sample and the cavity resonator, respectively.

The conductivity can be related to the imaginary part of the relative dielectric constant as:

$\sigma_{\mathrm{e}}=\omega \varepsilon^{\prime \prime}=2 \Pi f \varepsilon_{0} \varepsilon_{\mathrm{r}}^{\prime \prime}$

\section{Results and discussion}

The dielectric properties of ionomers at microwave frequencies are determined and tabulated in Table 1. It is found that as the zinc content in the HSR increases, the dielectric constant remains nearly the same but the imaginary part of the complex permittivity increases drastically. The variation of conductivity of the sample with frequency is shown in Fig. 4. Ionic polymer has numerous potential applications in plastic industry, package industry[6], electrophotography $[7,8]$ and dentistry [9]. Because of its high thermal stability, mechanical strength and high conductivity, it is superior to conducting polymer [3]. The microwave applications of ionomers are yet to be explored. The possibilities of using ionomers in microwave monolithic integrated circuits (MMIC) and microwave devices can be investigated.

\section{References}

[1] D.Y. Chao, J. Kuo, N.H. Wang, C.L. Lee, K.H. Yang, J. Appl. Polym. Sci. 67 (1998) 19.

[2] J.-W. Lee, C.-H. Kim, J.-K. Park, T.-S. Hwang, Polym. Int. 45 (1998) 47.

[3] J. Samuel, T. Xavier, T. Kurian, Prog. Rubber Plast. Technol. 16 (2000) 1 .

[4] K.T. Mathew, U. Raveendrannath, Sensors Update (Ger.) 7 (1999) 185

[5] U. Raveendranath, K.T. Mathew, J. Mol. Liq. 68 (1996) 145.

[6] W.J. MacKnight, R.D. Lundberg, Thermoplastic Elastomers (Munich), 1987.

[7] J.S. Tan, NATO ASI Series C: Mathematical and Physical Sciences, vol. 198, Reidel, Dordrecht, 1987, p. 439.

[8] A. Diaz, D. Fenzel-Alexander, D.C. Miller, D. Wollmann, A. Eisenberg, J. Polym. Sci., Part C: Polym. Lett. 28 (1990) 75.

[9] L. Stanislawski, E. Soheili-Majd, A. Perianin, M. Goldberg, J. Biomed. Mater. Res. 51 (2000) 469. 